\title{
A brain network instantiating approach and avoidance motivation
}

\author{
JEFFREY M. SPIELBERG, ${ }^{\mathrm{a}, \mathrm{b}}$ GREGORY A. MILLER, ${ }^{\mathrm{b}, \mathrm{c}, \mathrm{d}}$ STACIE L. WARREN, ${ }^{\mathrm{b}}$ ANNA S. ENGELS, ${ }^{\mathrm{b}, \mathrm{e}}$ \\ LAURA D. CROCKER, ${ }^{\mathrm{b}}$ MARIE T. BANICH,${ }^{\mathrm{f}}$ BRADLEY P. SUTTON, ${ }^{\mathrm{g}}$ AND WENDY HELLER ${ }^{\mathrm{b}}$ \\ ${ }^{a}$ Department of Public Health, University of California, Berkeley, Berkeley, California, USA \\ ${ }^{b}$ Department of Psychology, University of Illinois at Urbana-Champaign, Urbana, Illinois, USA \\ ${ }^{c}$ Department of Psychology, University of Delaware, Newark, Delaware, USA \\ dDepartment of Psychology, University of Konstanz, Konstanz, Germany \\ ${ }^{\mathrm{e}}$ Department of Psychology, Pennsylvania State University, University Park, Pennsylvania, USA \\ ${ }^{\mathrm{f}}$ Department of Psychology and Neuroscience, University of Colorado, Boulder, Boulder, Colorado, USA \\ ${ }^{g}$ Department of Bioengineering, University of Illinois at Urbana-Champaign, Urbana, Illinois, USA
}

\begin{abstract}
Research indicates that dorsolateral prefrontal cortex (DLPFC) is important for pursuing goals, and areas of DLPFC are differentially involved in approach and avoidance motivation. Given the complexity of the processes involved in goal pursuit, DLPFC is likely part of a network that includes orbitofrontal cortex (OFC), cingulate, amygdala, and basal ganglia. This hypothesis was tested with regard to one component of goal pursuit, the maintenance of goals in the face of distraction. Examination of connectivity with motivation-related areas of DLPFC supported the network hypothesis. Differential patterns of connectivity suggest a distinct role for DLPFC areas, with one involved in selecting approach goals, one in selecting avoidance goals, and one in selecting goal pursuit strategies. Finally, differences in trait motivation moderated connectivity between DLPFC and OFC, suggesting that this connectivity is important for instantiating motivation.
\end{abstract}

Descriptors: Motivation, Normal volunteers, fMRI/PET/MRI

A long line of research indicates that dorsolateral prefrontal cortex (DLPFC) is centrally involved in the pursuit of goals (e.g., MacDonald, Cohen, Stenger, \& Carter, 2000). One potential role for DLPFC is the integration of motivation and executive function necessary for goal pursuit. This hypothesis has been supported by research examining the integration of processes related to executive function and to approach and avoidance motivation in both state motivation (induced by the presence of immediate reward and/ or punishment; Taylor et al., 2004) and trait motivation (temperamental tendencies to be sensitive to, and motivated by, potential appetitive or aversive outcomes; Spielberg, Miller, et al., 2011; Spielberg et al., 2012). However, given the complexity of the processes involved in the pursuit of approach and avoidance goals, it is unlikely that DLPFC functions in isolation. Rather, DLPFC is likely to be part of a network of brain regions involved in instantiating motivational processes. However, this hypothesis has not been explicitly examined in the context of one important aspect of goal pursuit, namely, the maintenance of goals in the face of distraction. The aim of the present study was to test the hypothesis that

This work was supported in part by the National Institute of Mental Health (R01 MH61358, P50 MH079485, and T32 MH19554), the National Institute of Drug Abuse (R21 DA14111), and the Beckman Institute for Advanced Science and Technology of the University of Illinois at UrbanaChampaign. The authors thank Katie Mimnaugh, Kyle Gerst, and Naomi Sadeh for their assistance in the completion of this project.

Address correspondence to: Jeffrey M. Spielberg, Ph.D., University of California, Berkeley, 4135 Tolman Hall, Berkeley, CA 94720, USA. E-mail: jspielb2@gmail.com motivation-related regions of DLPFC are part of a network of brain areas involved in maintaining approach/avoidance goals. If DLPFC is part of such a network, connectivity between DLPFC and regions in the network should increase when the maintenance of a goal is challenged. Additionally, individuals with higher levels of approach/avoidance motivation should show larger increases in connectivity than those low in approach/avoidance motivation. Thus, the present study compared DLPFC connectivity when goal maintenance was challenged relative to when no such challenge was present and tested whether measures of trait motivation moderated the magnitude of changes in connectivity.

\section{A Network for Goal Pursuit}

Research supports the existence of two fundamental motivational systems, one oriented toward potential desirable outcomes, termed the approach motivational system, and one oriented toward potential aversive outcomes, termed the avoidance motivational system (for reviews, see Elliot \& Covington, 2001; Lang, Bradley, \& Cuthbert, 1998). Stable individual differences in the activity and/or reactivity of these motivational systems have been found, and these differences have been hypothesized to reflect distinct temperament types (Elliot \& Thrash, 2002). Recent research indicates that approach temperament, reflecting stable differences in the approach motivational system, is associated with activity of leftlateralized regions of DLPFC, and avoidance temperament, reflecting stable differences in the avoidance motivational system, is associated with activity of a right-lateralized region of DLPFC (Spielberg, Miller, et al., 2011; Spielberg et al., 2012). These 
findings suggest that left DLPFC is differentially associated with the pursuit of approach-related goals and right DLPFC with the pursuit of avoidance-related goals, which is consistent with a large body of research indicating that prefrontal cortex (PFC) is lateralized with respect to motivational direction and emotional valence (for a review, see Spielberg, Stewart, Levin, Miller, \& Heller, 2008). This conceptualization was refined further by research indicating that portions of left and right DLPFC are involved in approach and avoidance behavior independent of the valence of the stimuli involved (Berkman \& Lieberman, 2010), supporting the role of DLPFC in the pursuit of goals separate from valuation of stimuli. Additionally, evidence indicates that a region in left DLPFC is associated with both approach and avoidance temperament (Spielberg, Miller, et al., 2011; Spielberg et al., 2012), suggesting that some areas of DLPFC implement motivational processes that are not specific to approach or avoidance motivation (e.g., energization of behavior independent of motivational direction of behavior).

These findings are consistent with the proposal (Spielberg, Miller, et al., 2011) that regions of DLPFC integrate executive and motivational processes during goal pursuit, although the exact nature of these processes remains unclear. Given strong evidence of DLPFC involvement in maintaining and updating information (e.g., Wager \& Smith, 2003) and in top-down control (e.g., Dosenbach, Fair, Cohen, Schlaggar, \& Petersen, 2008), it is possible that these regions of DLPFC maintain and integrate information relevant to approach and avoidance goal pursuit and use this information to bias processing in other brain regions to be congruent with the pursuit of goals when the pursuit of goals is challenged.

Although Spielberg, Miller, et al. (2011) hypothesized that DLPFC has a central role in approach and avoidance goal pursuit, it is unlikely that DLPFC functions in isolation. Rather, DLPFC is likely to be part of a network, with each node having distinct roles in the pursuit of goals (Miller \& Cohen, 2001). Several recent studies have found that DLPFC exhibits connectivity with several brain regions thought to be important for goal pursuit and that this connectivity depends on the level of motivational incentive present. For example, Szatkowska, Bogorodzki, Wolak, Marchewka, and Szeszkowski (2008) found that DLPFC connectivity with orbitofrontal cortex (OFC) increased with the level of available reward during a working memory task, and a number of studies have found that DLPFC connectivity with portions of the basal ganglia (BG; e.g., nucleus accumbens, putamen) depends on the level of monetary reward (e.g., Ballard et al., 2011; Staudinger, Erk, \& Walter, 2011). Similarly, reward dependent connectivity between DLPFC and the dorsal aspect of anterior cingulate (ACC) has been observed when participants had to switch between task rules (Kouneiher, Charron, \& Koechlin, 2009). Evidence of anatomical connections between DLPFC and these regions (e.g., M. Beckmann, Johansen-Berg, \& Rushworth, 2009; Sakagami \& Watanabe, 2007) further supports the hypothesis that they function as a network.

Although this research supports the hypothesis that DLPFC functions as part of a network of regions during the pursuit of goals, research has yet to examine whether DLPFC similarly functions as part of such a network in the context of one vital aspect of goal pursuit, the maintenance of goal pursuit in the face of distraction. Given evidence that DLPFC is heavily involved in top-down control (Dosenbach et al., 2008), it is reasonable to expect DLPFC to play a vital role in moderating the activation of other brain regions involved in goal pursuit in order to maintain the pursuit of goals in the face of distraction. However, this hypothesis has yet to be directly tested, leaving open the question of DLPFC's role in a network during the maintenance of goals.

The present study tested this hypothesis by examining connectivity between DLPFC and several brain regions that appear to be integral to approach and avoidance goal pursuit. Specifically, the present study focused on OFC, ACC, amygdala, and BG. This set of regions is certainly only a subset of those involved in goal pursuit. The present study focused on this subset because evidence suggests that (a) these regions instantiate central aspects of approach and avoidance motivation (discussed briefly below) and (b) they are likely to be involved in the aspect of goal pursuit studied here (maintenance of goal pursuit in the face of distraction). There are a number of reviews discussing the potential roles of these regions in motivation and goal pursuit (e.g., Amiez \& Petrides, 2009; Berkman \& Lieberman, 2009; Cain \& LeDoux, 2008; Davidson \& Irwin, 1999; Porcelli \& Delgado, 2009; Rangel $\&$ Hare, 2010). Therefore, only a brief discussion of the role of these regions will be provided here, and interested readers are referred to these reviews for further information.

Orbitofrontal cortex. Research suggests that OFC is involved in maintaining the current and expected motivational value of stimuli (O'Doherty \& Dolan, 2006). Stimulus value information is vital to maintaining goals in the face of distraction because it is important in the determination of whether the distracting information indicates the presence of a more valuable goal. Research suggests that OFC provides information about stimulus value to DLPFC (Szatkowska et al., 2008), which could then be used to select appropriate goals. However, researchers have also hypothesized that DLPFC modulates stimulus values stored in OFC to be congruent with current goals (e.g., decreasing the value of liked but unhealthy foods in dieters; Hare, Camerer, \& Rangel, 2009). Thus, the relationship between DLPFC and OFC may be bidirectional, with stimulus value both informing and being informed by current goals.

The spatial organization of OFC remains a matter of debate. Specifically, some research supports a medial/lateral distinction, with medial and lateral OFC involved in maintaining reward values and punishment values, respectively (Elliott, Dolan, \& Frith, 2000; Kringelbach \& Rolls, 2004; O’Doherty, 2007). However, a medial/ lateral parcellation does not incorporate hemispheric laterality, and research suggests that hemispheric laterality is an important organizing factor for PFC with respect to motivation and emotion (Davidson \& Irwin, 1999; Heller, 1993). Wager et al. (2008) provided insight into how medial/lateral and hemispheric organizations of OFC may be integrated, finding that bilateral medial and right lateral OFC were associated with pleasant emotional experience, whereas left middle and left lateral OFC were associated with unpleasant emotional experience.

Anterior cingulate cortex. ACC is also thought to have a central role in the pursuit of goals (Rushworth, Behrens, Rudebeck, \& Walton, 2007). One popular theory holds that ACC is involved in the detection of error and/or conflict (e.g., Carter et al., 1998). However, ACC activation has also been observed in contexts without error or conflict, such as when detecting cues signaling potential reward (Bush et al., 2002). In an attempt to reconcile the extant evidence, Rushworth and Behrens (2008) proposed that ACC is involved in encoding the predicted value associated with actions, information that is necessary for creating efficient strategies for goal pursuit. Given that DLPFC has also been implicated in the selection of optimal action strategies (Frith, 2000), one 
possibility is that ACC and DLPFC interact to determine the strategy used to pursue goals. Supporting this hypothesis, a recent study (M. Beckmann et al., 2009) identified a region (roughly corresponding to what has been labeled dorsal ACC [Bush, Luu, \& Posner, 2000] and extending around the genu of the corpus callosum) that was reliably activated by reward manipulations and had abundant white-matter connections with DLPFC and surrounding cortex, suggesting that this ACC region provides motivational information regarding actions to DLPFC. Action value information appears to be vital to the maintenance of goals in the face of distraction, because it is important for determining whether the distracting information signals that the current action plan should be adjusted.

Amygdala. Amygdala is involved in identifying motivationally salient stimuli and enhancing perceptual processing of such stimuli (Pessoa \& Adolphs, 2010). These processes appear to be vital in maintaining goal pursuit in the face of distraction, because they are important in determining to which stimulus aspects to attend. Although traditionally discussed with regard to the identification of unpleasantly valenced stimuli (e.g., Adolphs, Tranel, Damasio, \& Damasio, 1995), amygdala is also involved in identifying pleasantly valenced stimuli (e.g., Holland \& Gallagher, 2004; Sabatinelli, Bradley, Fitzsimmons, \& Lang, 2005), and its engagement appears not to be dependent on valence per se.

Basal ganglia. Another set of regions thought to be important for the pursuit of goals is BG, which is made up of striatum, globus pallidus, substantia nigra, and subthalamic nucleus. Research implicates these subcortical nuclei in a number of motivational processes (Haber, 2009), such as anticipating rewarding stimuli (Knutson, Taylor, Kaufman, Peterson, \& Glover, 2005), reinforcing actions (Tricomi, Delgado, \& Fiez, 2004), and integrating motivational information from diverse areas and using this integrated information to moderate ongoing processing in target brain areas (e.g., OFC; Haber, 2009). These processes appear to be central to the maintenance of goals in the face of distraction because, for example, it is important to integrate stimulus and action values associated with the goal and with distracting information in order to influence behavior appropriately.

\section{The Present Study}

Although research suggests that all of the brain areas discussed above are important for goal pursuit, the hypothesis that these regions function as a network to maintain approach and avoidance goals in the face of distraction remains untested. The present study tested this hypothesis using functional magnetic resonance imaging (fMRI) while participants performed a task that manipulated distraction from goal pursuit, the color-word Stroop task (Stroop, 1935). When the maintenance of goal pursuit (naming of ink color) is challenged by distracting information (when word meaning and ink color are incongruent), connectivity should be higher between DLPFC and brain regions involved in the pursuit of goals than when no such distraction is present (when word meaning and ink color are congruent). The present investigation tested this hypothesis via analysis of psychophysiological interaction (PPI) between DLPFC activation and task condition (incongruent, congruent) predicting activity in the brain regions discussed above.

Although the PPI analyses can identify a network of brain regions that interact with motivation-related DLPFC regions to maintain goal pursuit, these analyses would not indicate the extent to which connectivity in this network specifically instantiates approach and avoidance motivation. If this network instantiates approach and avoidance motivation, individuals with higher levels of trait motivation should show greater connectivity. Therefore, three-way PPIs between DLPFC activation, task condition, and questionnaire measures of temperamental motivation were examined.

Three specific areas of DLPFC were examined in the present study based on a priori clusters identified by Spielberg, Miller, et al. (2011): a cluster in left DLPFC related to approach motivation, a cluster in right DLPFC related to avoidance motivation, and a cluster in left DLPFC related to both approach and avoidance motivation. Although all three DLPFC clusters were hypothesized to be involved in goal pursuit and thus should be connected with all the candidate network nodes, it is possible that the clusters have distinct roles in goal pursuit. If so, the DLPFC clusters should exhibit differential connectivity with the network nodes. Hypotheses related to differential connectivity with OFC were examined: Given evidence that medial and left lateral OFC are associated with maintaining reward and punishment value, respectively, the DLPFC cluster associated with approach motivation was hypothesized to exhibit greater connectivity with medial OFC than the DLPFC cluster associated with avoidance motivation, and vice versa for left lateral OFC. Additionally, both clusters associated with only one type of motivation were expected to show greater connectivity with OFC than the cluster associated with both approach and avoidance motivation, given that this cluster may be involved in processes that are independent of motivational direction.

The present study used two independent samples. The first sample was the data examined in Spielberg, Miller, et al. (2011). Because these data were used to identify the DLPFC seed clusters, it is possible that the findings could be biased by overfitting of the seed clusters. Specifically, cluster shape in Spielberg, Miller, et al. may have been partially determined by error variance shared with other areas of the brain. Therefore, a second, independent sample was used to replicate these analyses in order to rule out this potential confound. In addition, the second study tested generalizability across a wider demographic.

\section{Method}

\section{Participants}

Sample 1. Participants were the same sample of undergraduate students used in Spielberg, Miller, et al. (2011). The initial sample consisted of 107 participants. Data from 25 participants were not used because the participant (a) moved more than $3.3 \mathrm{~mm}$ (the smallest side of a voxel) relative to the volume used for registration (the middle volume of the time series) or more than $2 \mathrm{~mm}$ relative to the previous volume, (b) committed errors on $15 \%$ or more of the trials, (c) exhibited reaction times greater than 3 standard deviations from the mean, (d) exhibited apparent signal loss due to magnetic susceptibility in areas of interest, and/or (e) exhibited activation patterns that appeared to be due to residual motion artifact. The final sample consisted of 82 participants (57\% female, mean age $=19.1$ years). One participant's scans exhibited scanner artifact throughout the time series. Independent components analysis, as implemented in MELODIC (C. Beckmann \& Smith, 2004), was used to isolate and remove this artifact. After removal, no artifact was apparent. 
Sample 2. An unselected sample of participants was collected in order to test whether the findings for Sample 1 were generalizable to a broader sample. These participants were recruited from the community using advertisements placed in local newspapers and an electronic list-serve. Participant screening and data-quality procedures were identical to those used for Sample 1. A total of 120 participants completed the protocol, and data from 102 participants (63\% female, mean age $=34.2$ years $)$ passed data quality screening. All study procedures (e.g., task design, data collection) were identical across samples.

Combined sample. Given that fMRI data are relatively noisy (Lazar, 2008) and thus have low reliability, the magnitude of the observed relationships between brain regions would be attenuated (Bollen, 1989). Therefore, the power to detect differences among the three DLPFC clusters in connectivity with nodes in the proposed network would be reduced. Additionally, low reliability would lead to a restriction in the range of levels of connectivity with DLPFC, reducing the power to detect moderation of such connectivity by temperamental motivation. Therefore, Samples 1 and 2 were combined (total $N=184$ ) in order to test whether connectivity differed among the DLPFC clusters and whether connectivity with DLPFC clusters was moderated by temperamental motivation. Data from eight participants were not used in the moderation analyses because of missing questionnaire data $(N=176$, $60.2 \%$ female, mean age $=27.3$ years $)$.

\section{Questionnaires}

As was done in Spielberg, Miller, et al. (2011), three questionnaires that have been associated with Approach and Avoidance Temperament were administered to measure these constructs (Elliot \& Thrash, 2002; Spielberg, Heller, Silton, Stewart, \& Miller, 2011): the Behavioral Activation and Behavioral Inhibition Scales (Carver \& White, 1994), the Extraversion and Neuroticism subscales of the NEO-Five Factor Inventory (Costa \& McRae, 1992), and the Positive and Negative Temperament subscales of the General Temperament Survey (Watson \& Clark, 1993).

\section{DLPFC Seed Clusters}

The DLPFC seed areas were those identified by Spielberg, Miller, et al. (2011). Participants in the Spielberg, Miller, et al. study performed the color-word Stroop, and analyses identified regions in which activation during the maintenance of goal pursuit was greater for those individuals who were higher on trait approach or avoidance motivation or both. One cluster was located in left DLPFC (BA 9, max z MNI coordinates $=[-36,30,46])$ and was associated with approach temperament, and one cluster was located in right DLPFC (BA 9/8/6, max z MNI coordinates $=[36,12,44])$ and was associated with avoidance temperament. Additionally, one cluster in left DLPFC (BA 9/8) was examined in the present study that merged two overlapping clusters associated with approach $(\max \mathrm{z}$ MNI coordinates $=[-18,46,36])$ and avoidance $(\max \mathrm{z}$ MNI coordinates $=[-8,42,36])$ given that this area may implement motivational processes not specific to approach or avoidance motivation. This combined cluster was anterior to the cluster associated with only approach motivation. Thus, three DLPFC clusters were used: (a) one in left DLPFC related to approach temperament, (b) one in right DLPFC related to avoidance temperament, and (c) one in left DLPFC related to both approach and avoidance.

\section{Stimuli and Experimental Design}

Participants completed two tasks, a color-word Stroop and an emotion-word Stroop (duration of each task $=12 \mathrm{~min} 20 \mathrm{~s}$ ) in fMRI and electroencephalography (EEG) sessions (findings from the emotion-word Stroop tasks and EEG sessions are not presented here). The order of presentation of the two tasks and the two sessions was counterbalanced across participants. In the colorword Stroop task, blocks of color-congruent or color-incongruent words alternated with blocks of neutral words. Additional neutral trials were intermixed 50:50 in congruent and incongruent blocks to prevent the development of word-reading strategies. This type of blocked-design color-word Stroop task has been shown to effectively elicit Stroop interference (Banich et al., 2000; Compton et al., 2003). The order of presentation of blocks in the present investigation was counterbalanced for each participant. In addition to the word blocks, there were four fixation blocks (one at the beginning, one at the end, and two in the middle of the session) and five rest blocks (one at the beginning, one at the end, and one between each word block).

The task consisted of 256 trials presented in 16 blocks (four congruent, four incongruent, and eight neutral) of 16 trials each, with a variable intertrial inteval (ITI; $2000 \pm 225 \mathrm{~ms}$ ) between trial onsets. A trial began with presentation of a word for $1500 \mathrm{~ms}$, followed by a fixation cross for an average of $500 \mathrm{~ms}$. Each trial consisted of one word presented in one of four ink colors (red, yellow, green, blue), each color occurring equally often with each word type. The task consisted of congruent trials in which the word named the ink color in which it was printed (e.g., the word "RED" printed in red ink), incongruent trials in which the word named a color incongruent with the ink color in which it was printed (e.g., "GREEN" in red ink), and neutral trials in which the word was unrelated to color (e.g., "LOT" in red ink). Neutral words were matched with color words for word frequency and length. Each word (visual angle $6-16^{\circ}$ ) was centered on a black background and projected.

\section{fMRI Data Collection}

The fMRI data were 370 three-dimensional (3D) images acquired using a Siemens gradient-echo echo-planar imaging sequence (TR $2000 \mathrm{~ms}$, TE $25 \mathrm{~ms}$, flip angle $80^{\circ}, \mathrm{FOV}=22 \mathrm{~cm}$ ) on a Siemens Allegra 3T scanner. Each image consisted of 38 oblique axial slices (slice thickness $3 \mathrm{~mm}, 0.3-\mathrm{mm}$ gap, in-plane resolution $3.4375 \times 3.4375 \mathrm{~mm}$ ) acquired parallel to the anterior and posterior commissures. After the fMRI acquisition, a 160-slice MPRAGE structural sequence was acquired (spatial resolution $1 \mathrm{~mm} \times 1 \mathrm{~mm} \times 1 \mathrm{~mm}$ ) and used to warp the participant's functional data into standard space.

\section{fMRI Data Reduction and Preprocessing}

Image processing and statistical analysis were implemented primarily using FEAT v5.98, part of the FSL analysis package (http://www.fmrib.ox.ac.uk/fsl). The first three time points (fMRI volumes) of the data set corresponding to each task for each subject were discarded to allow the MR signal to reach a steady state. Functional data for each participant were motion corrected using the MCFLIRT linear registration tool (Jenkinson, Bannister, Brady, \& Smith, 2003), intensity normalized, temporally filtered with a nonlinear high-pass filter, and spatially smoothed using a 3D 
Gaussian kernel (FWHM $=5 \mathrm{~mm}$ ). Temporal low-pass filtering was carried out using AFNI's 3dDespike tool (http://afni. nimh.nih.gov/).

\section{fMRI Data Processing}

Psychophysiological interaction analyses were performed on the preprocessed functional time series of each participant using FILM (Woolrich, Ripley, Brady, \& Smith, 2001). For each participant, a separate analysis was conducted for each of the three DLPFC regions of interest (ROIs). For each DLPFC ROI, the DLPFC ROI was warped from standard space into the participant's functional space, and a predictor was created by extracting the mean value across all voxels in the ROI for each of the 370 time points. In each analysis, six predictors were entered: (1) one of the DLPFC time series predictors, (2) a congruence predictor (IvC) that modeled the difference between incongruent and congruent conditions (coded as 1 during the incongruent condition, -1 during the congruent condition, and 0 at all other times), (3) the interaction (represented by the product) of the DLPFC time series and congruence predictors, and (4-6) three predictors of no interest that modeled the variance associated with the sum of the incongruent and congruent conditions (in order to model the variance shared among these conditions), the neutral condition, and the rest condition. The IvC predictor and the three predictors of no interest were convolved with a gamma function to better approximate the temporal course of the BOLD hemodynamic response function (this convolution was performed on the $\mathrm{IvC}$ predictor prior to creating the interaction term). Each predictor yielded a per-voxel effect-size parameter estimate $(\beta)$ map representing the magnitude of activation associated with that predictor. For each participant, these functional activation maps, as well as the corresponding structural MRI map, were warped into a common stereotaxic space (MNI 152 symmetrical $1 \mathrm{~mm} \times 1 \mathrm{~mm} \times 1 \mathrm{~mm}$ template; Fonov, Evans, McKinstry, Almli, \& Collins, 2009) using FNIRT (Andersson, Jenkinson, \& Smith, 2007).

Group inferential statistical analyses were carried out using FLAME (FMRIB's Local Analysis of Mixed Effects). The $\beta$ maps corresponding to the interaction terms were entered as dependent variables into separate one-sample, two-tailed $t$ tests. Each $t$ test produced one $\beta$ map that corresponded to the mean of the interaction across the sample. The $t$ tests were then converted to $z$ scores to determine the significance of the $\beta$ s.

Monte Carlo simulations via AFNI's AlphaSim program were used to estimate the overall significance level for thresholding the 3D functional $z$ map image (Ward, 2000). These simulations provided the appropriate cluster size, which, in combination with an individual voxel $z$ threshold of $p=.04$, gave an overall two-tailed family-wise error rate of .05. Four masks of a priori regions of interest were used to limit the number of voxels under consideration. These masks were of (a) ventral prefrontal cortex (including OFC; cluster threshold $=897 \mathrm{~mm}^{3}$ ), (b) cingulate and paracingulate gyri (cluster threshold $=819 \mathrm{~mm}^{3}$ ), (c) amygdala (cluster threshold $=351 \mathrm{~mm}^{3}$ ), and (d) striatum and globus pallidus (cluster threshold $=585 \mathrm{~mm}^{3}$ ). These masks were created using the Harvard/Oxford probabilistic atlases that come with FSL (thresholded at 15\%). Because we used a standard space image that is slightly different from the one used to create these masks, we registered the standard image used in the creation of the atlases to the standard image used in the present study and applied this registration to the masks (followed by binarization). After warping, the masks appeared to accurately map the relevant regions.

\section{Confound Detection Analyses}

An additional set of analyses was conducted in order to rule out the potential confound that the findings for a given DLPFC cluster were being driven by shared variance with the other two DLPFC clusters rather than variance specific to that cluster. Specifically, analyses were rerun with new DLPFC predictors (and associated interaction predictors) that captured only the unique variance associated with that DLPFC cluster. These predictors were created for each participant, for each DLPFC cluster, by partialling out the variance associated with the other two DLPFC predictors prior to creating the interaction term. In addition to the new DLPFC and interaction predictors and the other predictors included in the original analyses (IvC and the predictors of no interest), two predictors were included in each analysis. These were the predictors corresponding to the time series from the other two DLPFC clusters and were included so that the shared variance, which was removed from the new DLPFC predictor, would also be removed from the error term to ensure that the tests were not biased toward Type II errors. To ensure that the inclusion of these two predictors did not interfere with the test of the interaction, the variance associated with the interaction was partialled out of the two predictors before entering them into the model. These analyses were thresholded in the same manner as the original analyses described above, except that onetailed $t$ tests were used, given that the direction of the effect of interest was specified by the findings of the original analysis.

\section{Interaction Decomposition}

For each DLPFC cluster, the interaction analysis identifies voxels in other brain regions with time series that show significantly different correlations with DLPFC depending on the task condition (incongruent vs. congruent). Thus, the interaction analysis tests whether there is a significant difference in correlations between conditions and can also indicate the sign of that difference (e.g., the correlation is more positive during incongruent than congruent). However, these analyses cannot provide the size or sign of the individual correlations for each condition, which limits the interpretations that can be made about the relationships.

Therefore, analyses were conducted to determine the size and sign of the individual correlations for each condition. For each cluster that emerged from an interaction analysis, the time series data for that cluster were extracted separately for the incongruent and congruent blocks and regressed (separately for incongruent and congruent) on the relevant DLPFC seed cluster time series. Only time points corresponding to when the convolved congruence predictor had reached its maximum absolute value were used, leaving 12 time points per block. These regressions were conducted using the Mixed procedure in SPSS version 18. Participant was the nesting variable, and block and time point were repeated factors. The level 1 covariance matrix was modeled with a lag 1 autoregressive function. Regression $\beta$ s were converted into correlations using the $t$ value and degrees of freedom corresponding to that $\beta$. Specifically, the $t$ value was divided by the square root of the sum of the degrees of freedom and the squared $t$ value.

\section{Conjunction Analyses}

A conjunction analysis was carried out for connectivity with each DLPFC cluster using the method outlined by Nichols, Brett, Andersson, Wager, and Poline (2005). Specifically, a conjunction $z$ map was created by taking the least significant $z$ value (the $z$ value 
closest to 0 ) for each voxel from the $z$ maps for the two samples (if the $z$ values differed in sign, a value of 0 was assigned instead). For example, if the $z$ values at voxel $[1,1,1]$ for Sample 1 and Sample 2 were 1.6 and 1.9 , respectively, the value of voxel $[1,1,1]$ in the conjunction map would be the Sample 1 value (1.6). The conjunction maps were then thresholded in the manner described above (using the same masks), except one-tailed tests were used, given that all effects in both samples were in the positive direction.

\section{Connectivity Comparisons}

To determine whether the DLPFC clusters had differential patterns of connectivity, three fixed-effects, voxel-wise paired $t$ tests were conducted that compared the $\beta$ s corresponding to connectivity between one DLPFC cluster and a voxel to the $\beta$ s corresponding to connectivity between another DLPFC cluster and that voxel. These analyses used the PPI analyses in which the variance associated with the other two DLPFC clusters had been partialled out in order to maximize the specificity of the findings. These analyses were thresholded in the manner described above. One-tailed tests were used for comparisons in which a specific a priori directional difference was hypothesized. Additionally, masks of medial (cluster threshold $=663 \mathrm{~mm}^{3}$ ) and lateral (cluster threshold $=663 \mathrm{~mm}^{3}$ ) OFC were used for the comparison between the DLPFC cluster associated with only approach and the cluster associated only with avoidance.

\section{Moderation of PPI Connectivity by Temperamental Motivation}

Computation of approach and avoidance temperament scores. Approach and avoidance factor scores were computed using the methods reported by Spielberg, Heller, et al. (2011), Spielberg, Miller, et al. (2011), and Spielberg et al. (2012). Specifically, the questionnaire scales were used as indicators in confirmatory factor analysis using SPSS's AMOS. Two latent factors were modeled, with Behavioral Activation, Extraversion, and Positive Temperament used as indicators for approach temperament and Behavioral Inhibition, Neuroticism, and Negative Temperament used as indicators for avoidance temperament. Maximum likelihood estimation was used, and the two latent factors were allowed to covary freely. Factor scores were extracted with the regression method to use as measures of approach and avoidance temperament. These procedures closely follow those established by Elliot and Thrash (2002), who also observed the factor structure observed in the present study and found these factors to be linked systematically to approach and avoidance goal pursuit.

Data analysis. To assess moderation of PPI connectivity by temperamental motivation, the PPI $\beta$ maps computed earlier were entered as dependent variables in a higher level regression. Approach and avoidance factor scores were entered as predictors of PPI connectivity with the left DLPFC cluster associated only with approach and the right DLPFC cluster associated only with avoidance. Given that the left DLPFC cluster associated with both approach and avoidance is hypothesized to be involved in processes independent of motivational direction, the sum of the approach and avoidance factors scores was used to predict PPI connectivity for this cluster. Participant age and gender were included as covariates of no interest to account for error variance. These analyses were thresholded in the manner described above.

Approach temperament was hypothesized to be associated with increased connectivity between the approach-related left DLPFC cluster and network nodes, avoidance temperament was hypothesized to be associated with increased connectivity between the avoidance-related right DLPFC cluster and network nodes, and the approach/avoidance sum score was hypothesized to be associated with increased connectivity between the approach/avoidancerelated left DLPFC cluster and network nodes. Given these a priori directional hypotheses, one-tailed tests were used for these comparisons. To provide more specific tests, masks of anteriormedial (cluster threshold $=663 \mathrm{~mm}^{3}$ ) and right agranular/lateral OFC (cluster threshold $=624 \mathrm{~mm}^{3}$ ) were used for moderation by approach temperament of the left DLPFC cluster associated only with approach, given that these areas exhibited consistent connectivity with this DLPFC cluster. Similarly, masks of anterior-medial and lateral OFC were used for moderation by avoidance temperament of the right DLPFC cluster associated only with avoidance.

\section{Results}

\section{Sample 1}

Table 1 lists brain regions that evidenced stronger positive correlations with activation in a DLPFC cluster during incongruent than congruent conditions. No clusters emerged in which the opposite pattern held. Table 1 also provides correlations between the identified brain regions and activation in this DLPFC cluster for each condition. All clusters exhibited larger positive correlations with DLPFC activation during incongruent blocks than during congruent blocks.

Approach temperament cluster in left DLPFC. The first set of analyses used the left DLPFC region found to be selectively associated with approach temperament in Spielberg, Miller, et al. (2011) as a seed cluster. Three clusters emerged in OFC (Figure 1A), one each in right agranular/lateral OFC, in left agranular OFC/posteriormiddle OFC, and in medial-anterior OFC. One cluster emerged in cingulate located in dorsal ACC (dACC)/genual ACC (gACC)/ subgenual ACC/paracingulate gyrus (Figure 1B). Two clusters emerged in bilateral amygdala (Figure 1C). Three clusters emerged in BG (Figure 1D), two in bilateral putamen/globus pallidus, and one in right caudate. All clusters remained significant when only the unique variance associated with the seed cluster was used, indicating that these findings are not due to variance shared with other seed clusters.

Avoidance temperament cluster in right DLPFC. The second set of analyses used the right DLPFC region found to be selectively associated with avoidance temperament in Spielberg, Miller, et al. (2011) as a seed cluster. As illustrated in Figure 1E, two clusters emerged in OFC, one in left agranular and posteriormiddle OFC, and one in medial-anterior OFC/frontal pole. Two clusters emerged in cingulate (Figure $1 \mathrm{~F}$ ). One large cluster was located in $\mathrm{dACC} / \mathrm{gACC} /$ subgenual $\mathrm{ACC} /$ paracingulate gyrus, with the other in posterior cingulate (PCC), extending into precuneus. One cluster emerged in left amygdala (Figure 1G) and three in BG (Figure 1H). One cluster was located in right nucleus accumbens/ putamen/globus pallidus, one in left putamen, and one in right caudate. With the exception of the amygdala cluster, all clusters remained significant when only the unique variance associated with the seed cluster was tested, indicating that these findings are not due to variance shared with the other DLPFC seed clusters. 
Table 1. Areas Exhibiting Condition-Dependent Correlations with DLPFC Clusters in Sample 1

\begin{tabular}{|c|c|c|c|c|c|c|c|c|}
\hline \multirow[b]{2}{*}{ Region } & \multirow[b]{2}{*}{ Cluster size $\left(\mathrm{mm}^{3}\right)$} & \multirow[b]{2}{*}{ Mean $z$ value } & \multirow[b]{2}{*}{ Cohen's $d$} & \multicolumn{3}{|c|}{ Location } & \multirow[b]{2}{*}{ Inc $\mathrm{r}$} & \multirow[b]{2}{*}{ Con $\mathrm{r}$} \\
\hline & & & & $\mathrm{X}$ & $\mathrm{Y}$ & $\mathrm{Z}$ & & \\
\hline \multicolumn{9}{|l|}{ L DLPFC associated with approach temperament } \\
\hline $\mathrm{M}$ anterior OFC (BA 11) & 1,625 & 2.50 & .57 & 3 & 52 & -23 & .08 & -.01 \\
\hline L agranular OFC/posterior-middle OFC (BA 11/47) & 4,163 & 2.49 & .57 & -30 & 9 & -20 & .20 & .10 \\
\hline $\mathrm{R}$ agranular/lateral OFC (BA 11/47) & 2,429 & 2.35 & .52 & 21 & 5 & -18 & .17 & .07 \\
\hline $\begin{array}{l}\text { M dACC/gACC/subgenual ACC/paracingulate gyrus } \\
\text { (BA 6/9/24/32/33) }\end{array}$ & 7,692 & 2.39 & .54 & 0 & 21 & 28 & .22 & .12 \\
\hline L amygdala & 1,217 & 2.69 & 61 & -19 & -2 & -15 & .13 & .01 \\
\hline $\mathrm{R}$ amygdala & 535 & 2.36 & .54 & 22 & 3 & -18 & .08 & .03 \\
\hline L putamen/globus pallidus & 3,158 & 2.45 & .56 & -28 & -19 & 5 & .14 & .06 \\
\hline $\mathrm{R}$ putamen/globus pallidus & 1,440 & 2.39 & .54 & 28 & -2 & -2 & .13 & .05 \\
\hline $\mathrm{R}$ caudate & 767 & 2.46 & .56 & 9 & 0 & 12 & .13 & .08 \\
\hline \multicolumn{9}{|l|}{ R DLPFC associated with avoidance temperament } \\
\hline M anterior OFC/frontal pole (BA 10) & 2,322 & 2.39 & .54 & 2 & 67 & 4 & .14 & .07 \\
\hline L agranular OFC/posterior-middle OFC (BA 11/47) & 2,911 & 2.49 & .57 & -47 & 19 & -8 & .21 & .12 \\
\hline $\begin{array}{l}\text { M dACC/gACC/subgenual ACC/paracingulate/PCC } \\
\text { (BA 6/9/23/24/31/32/33) }\end{array}$ & 20,674 & 2.56 & .58 & 1 & 42 & 0 & .30 & .22 \\
\hline M PCC/precuneus (BA 7/31) & 2,782 & 2.44 & .55 & -6 & -54 & 23 & .30 & .16 \\
\hline L amygdala ${ }^{\mathrm{a}}$ & 381 & 2.50 & .57 & -17 & -1 & -24 & .11 & .01 \\
\hline L putamen & 1,011 & 2.45 & .56 & -33 & -15 & -3 & .21 & .14 \\
\hline R NAc/putamen/globus pallidus & 5,103 & 2.53 & .58 & 22 & -5 & -8 & .29 & .17 \\
\hline $\mathrm{R}$ caudate & 1,271 & 2.39 & .54 & 15 & 10 & 13 & .24 & .18 \\
\hline \multicolumn{9}{|l|}{ L DLPFC associated with approach and avoidance temperament } \\
\hline $\mathrm{R}$ anterior-middle OFC (BA 10/11) & 1,303 & 2.50 & .57 & 31 & 65 & -6 & .19 & .10 \\
\hline $\mathrm{R}$ agranular OFC/IFG/insula (BA 11/13/47) & 3,215 & 2.47 & .56 & 47 & 23 & -10 & .27 & .19 \\
\hline M dACC/gACC/paracingulate gyrus (BA 6/24/32/33) & 11,666 & 2.56 & .58 & 8 & 25 & 28 & .41 & .33 \\
\hline M PCC (BA 23/31) & 1,032 & 2.31 & .52 & 2 & -37 & 24 & .31 & .22 \\
\hline M PCC/precuneus (BA 7/31) & 1,807 & 2.53 & .58 & 6 & -50 & 45 & .37 & .26 \\
\hline L amygdala & 622 & 2.68 & .61 & -18 & 1 & -22 & .23 & .12 \\
\hline $\mathrm{R}$ putamen/globus pallidus $\mathrm{a}^{\mathrm{a}}$ & 840 & 2.29 & .52 & 11 & -6 & -5 & .23 & .10 \\
\hline $\mathrm{R}$ caudate & 1,146 & 2.86 & .65 & 13 & 6 & 12 & .32 & .24 \\
\hline
\end{tabular}

Note. $\mathrm{L}=$ left. $\mathrm{R}=$ right. $\mathrm{M}=$ medial. $\mathrm{OFC}=$ orbitofrontal cortex. $\mathrm{IFG}=$ inferior frontal gyrus. $\mathrm{ACC}=$ anterior cingulate cortex. $\mathrm{dACC}=$ dorsal $\mathrm{ACC}$. $\mathrm{gACC}=$ genual ACC. $\mathrm{PCC}=$ posterior cingulate cortex. NAc = nucleus accumbens. $\mathrm{BA}=$ Brodmann's Area. Location: coordinates are for the maximum $z$ value and are for MNI152 2009 space, with the $x$ axis moving from left to right. Inc $\mathrm{r}=$ correlation with DLPFC seed cluster time series during the incongruent condition.

Con $\mathrm{r}=$ correlation with DLPFC seed cluster time series during the congruent condition.

${ }^{\text {a }}$ Cluster did not survive when only using the unique variance associated with the seed cluster.

Overlapping approach and avoidance cluster in left DLPFC. The third set of analyses used the left DLPFC region found to be associated with both approach and avoidance temperament in Spielberg, Miller, et al. (2011) as a seed cluster. As illustrated in Figure 1I, two clusters emerged in OFC. One cluster was located in right agranular OFC extending into inferior frontal gyrus (IFG) and insula, and the second cluster was located in right anterior-middle OFC. Three clusters emerged in cingulate, as illustrated in Figure 1J. One large cluster was located in dACC/gACC/ paracingulate gyrus. Two clusters emerged in PCC, one of which extended into precuneus. One cluster emerged in left amygdala (Figure 1K) and two in BG (Figure 1L). One cluster was located in right putamen/globus pallidus, and the other cluster was located in right caudate. With the exception of the putamen/globus pallidus cluster, all clusters remained significant when only the unique variance associated with the seed cluster was used, indicating that these findings are not due to the variance shared with other DLPFC seed clusters.

\section{Sample 2}

Table 2 lists brain regions that evidenced stronger positive correlations with a DLPFC cluster during the incongruent condition than congruent condition in the independent replication sample. No clusters emerged in which the opposite pattern held. Table 2 also provides the correlations between the identified brain regions and activation in this DLPFC cluster for each condition (incongruent, congruent). All clusters exhibited larger positive correlations with DLPFC activation during incongruent blocks than during congruent blocks.

Approach temperament cluster in left DLPFC. Replicating the findings for Sample 1, clusters were observed in right agranular/ lateral OFC and medial-anterior OFC (Figure 2A), dACC/ paracingulate gyrus and gACC (Figure 2B), right amygdala (Figure 2C), and bilateral putamen/globus pallidus and right caudate (Figure 2D). The Sample 1 clusters observed in left agranular OFC cluster and subgenual ACC were not replicated. Additionally, a cluster emerged in PCC, which was not evident in the Sample 1 analyses.

Several of the clusters observed in the Sample 2 did not remain significant when only the unique variance associated with the DLPFC seed cluster was used. These included the clusters in subgenual ACC and right caudate. Additionally, the clusters in medialanterior OFC and PCC did not remain significant, although these clusters were evident when an individual $z$ threshold of $p=.05$ (corrected for multiple comparisons) was used, suggesting that these effects are present, albeit weak. 

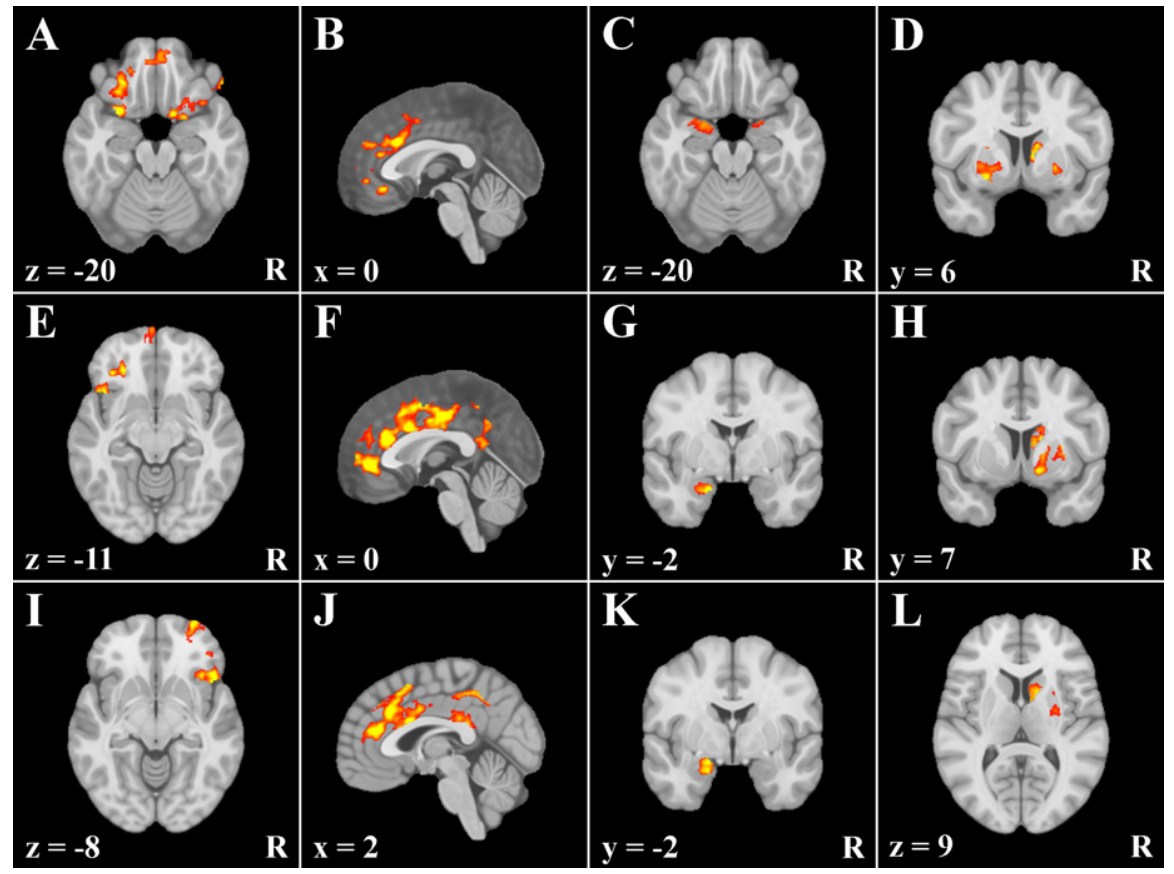

Figure 1. Areas exhibiting condition dependent correlations with DLPFC clusters in sample 1. R = right. $x, y$, and $z=$ coordinates in MNI 2009a space. A-D = clusters exhibiting greater connectivity with the left DLPFC cluster associated with approach temperament. E-H $=$ clusters exhibiting greater connectivity with the right DLPFC cluster associated with avoidance temperament. I-L = clusters exhibiting greater connectivity with the left DLPFC cluster associated with approach and avoidance temperament.

Avoidance temperament cluster in right DLPFC. Replicating the findings for Sample 1, clusters were observed in left agranular $\mathrm{OFC}$ and medial anterior $\mathrm{OFC} /$ frontal pole (Figure 2E), ACC/ gACC/paracingulate gyrus and PCC/precuneus (Figure $2 \mathrm{~F}$ ), and left putamen and right putamen/globus pallidus (Figure $2 \mathrm{G}$ ). The Sample 1 clusters observed in subgenual ACC, left amygdala, and right caudate were not replicated. Additionally, clusters emerged in right agranular OFC, left caudate, and left nucleus accumbens, which were not evident in the Sample 1 analyses. With the exception of the clusters in right putamen/globus pallidus and left caudate, all clusters remained significant when only the unique variance associated with the DLPFC seed cluster was used.

Left DLPFC associated with both approach and avoidance temperament. All regions observed in Sample 1 were replicated. Specifically, clusters were observed in right anterior-middle OFC and right agranular OFC/IFG/insula (Figure 2H), dACC/ gACC/paracingulate/PCC/precuneus (Figure 2I), left amygdala (Figure 2J), and right putamen/globus pallidus and right caudate (Figure 2K). Additionally, clusters emerged in left anterior-middle OFC, left agranular OFC/IFG/insula, right amygdala, left putamen/ globus pallidus, and left caudate, which were not evident in the Sample 1 analyses. All clusters remained significant when only the unique variance associated with the DLPFC cluster was used.

\section{Conjunction Analysis}

Table 3 lists brain regions that evidenced significant effects across both samples in the conjunction analysis.

Approach temperament cluster in left DLPFC. Clusters were observed in right agranular/lateral/OFC, dACC/paracingulate, right amygdala, left putamen/globus pallidus, and right putamen. Although clusters in medial-anterior OFC and right caudate were observed in both samples, they did not overlap enough to survive the conjunction analysis.

Avoidance temperament cluster in right DLPFC. Clusters were observed in left agranular OFC, dACC/gACC/paracingulate, PCC/ precuneus, and right putamen/globus pallidus. Although clusters in medial-anterior OFC/frontal pole and left putamen were observed in both samples, they did not overlap enough to survive the conjunction analysis.

Left DLPFC associated with both approach and avoidance temperament. Clusters were observed in right agranular $\mathrm{OFC} /$ IFG/insula, dACC/gACC/paracingulate, PCC/precuneus, left amygdala, and right putamen/globus pallidus/caudate. Although a cluster in right anterior-middle OFC was observed in both samples, it did not overlap enough to survive the conjunction analysis.

\section{Combined Sample}

Connectivity comparisons. Table 4 lists regions exhibiting differential task-condition-dependent connectivity with DLPFC clusters. Both DLPFC clusters associated only with one type of motivation exhibited stronger condition-dependent connectivity with anteriormedial OFC than did the DLPFC cluster associated with both temperament types. Additionally, the cluster associated only with approach exhibited greater condition-dependent connectivity with left agranular/posterior-middle OFC than did the cluster associated with both temperament types. The cluster associated only with avoidance exhibited greater condition-dependent connectivity with left lateral OFC than did the cluster associated with both 
Table 2. Areas Exhibiting Condition-Dependent Correlations with DLPFC Clusters in Sample 2

\begin{tabular}{|c|c|c|c|c|c|c|c|c|}
\hline \multirow[b]{2}{*}{ Region } & \multirow[b]{2}{*}{ Cluster size $\left(\mathrm{mm}^{3}\right)$} & \multirow[b]{2}{*}{ Mean $z$ value } & \multirow[b]{2}{*}{ Cohen's $d$} & \multicolumn{3}{|c|}{ Location } & \multirow[b]{2}{*}{ Inc $\mathrm{r}$} & \multirow[b]{2}{*}{ Con $\mathrm{r}$} \\
\hline & & & & $\mathrm{X}$ & $\mathrm{Y}$ & $\mathrm{Z}$ & & \\
\hline \multicolumn{9}{|l|}{ L DLPFC associated with approach temperament } \\
\hline $\mathrm{M}$ anterior $\mathrm{OFC}^{\mathrm{a}}(\mathrm{BA} 10 / 11)$ & 1,186 & 2.37 & .50 & 1 & 56 & -14 & .09 & .00 \\
\hline R agranular/lateral OFC (BA 11/47) & 1,156 & 2.52 & .51 & 38 & 25 & -16 & .08 & .01 \\
\hline M dACC/paracingulate (BA 6/23/24/32) & 5,785 & 2.58 & .52 & -2 & 5 & 39 & .15 & .04 \\
\hline $\mathrm{MgACC}{ }^{\mathrm{a}}(\mathrm{BA} 32)$ & 1,120 & 2.30 & .46 & 6 & 50 & -1 & .13 & .07 \\
\hline M PCC ${ }^{\mathrm{a}}(\mathrm{BA} 29 / 31)$ & 1,918 & 2.34 & .47 & 2 & -47 & 36 & .18 & .06 \\
\hline $\mathrm{R}$ amygdala & 730 & 2.69 & .55 & 27 & -1 & -15 & .10 & .01 \\
\hline L putamen/globus pallidus & 2,078 & 2.44 & .49 & -15 & 0 & 6 & .14 & .05 \\
\hline R NAc/caudate/putamen/globus pallidus & 3,889 & 2.42 & .49 & 12 & -2 & -1 & .15 & .02 \\
\hline R caudate ${ }^{\mathrm{a}}$ & 741 & 2.65 & .54 & 16 & 4 & 21 & .12 & .04 \\
\hline \multicolumn{9}{|l|}{ R DLPFC associated with avoidance temperament } \\
\hline L/M anterior OFC/frontal pole (BA 10/11) & 1,433 & 2.54 & .51 & -9 & 68 & -13 & .11 & .02 \\
\hline L agranular OFC (BA 11/47) & 2,274 & 2.41 & .49 & -36 & 25 & -20 & .22 & .13 \\
\hline R agranular OFC (BA 11/47) & 1,221 & 2.85 & .58 & 43 & 17 & -11 & .27 & .17 \\
\hline M dACC/gACC/paracingulate (BA 6/9/24/32) & 7,193 & 2.44 & .49 & -7 & 20 & 40 & .37 & .25 \\
\hline M PCC/precuneus (BA $7 / 23 / 29 / 31$ ) & 9,749 & 2.63 & .53 & -8 & -49 & 30 & .36 & .27 \\
\hline L putamen & 696 & 2.33 & .47 & -31 & 6 & 6 & .20 & .09 \\
\hline $\mathrm{R}$ putamen/globus pallidus ${ }^{\mathrm{a}}$ & 1,385 & 2.27 & .46 & 32 & 3 & 5 & .27 & .16 \\
\hline L caudate ${ }^{\mathrm{a}}$ & 608 & 2.44 & .49 & -15 & -13 & 22 & .29 & .19 \\
\hline L NAc & 615 & 2.36 & .48 & -13 & 13 & -5 & .21 & .13 \\
\hline \multicolumn{9}{|l|}{ L DLPFC associated with approach and avoidance temperament } \\
\hline $\mathrm{L}$ anterior-middle OFC (BA 10) & 1,112 & 2.45 & .50 & -40 & 51 & 3 & .33 & .23 \\
\hline $\mathrm{R}$ anterior-middle OFC (BA 10/11) & 4,696 & 2.56 & .52 & 28 & 53 & -12 & .29 & .18 \\
\hline L agranular OFC/IFG/insula (BA 11/13/47) & 1,740 & 2.51 & .51 & -29 & 28 & -6 & .33 & .23 \\
\hline $\mathrm{R}$ agranular OFC/IFG/insula (BA 11/13/47) & 4,396 & 2.69 & .55 & 49 & 21 & -2 & .30 & .18 \\
\hline $\begin{array}{l}\text { M dACC/gACC/subgenual ACC/ paracingulate gyrus/PCC/precuneus } \\
\text { (BA 6/7/9/23/24/29/31/32/33) }\end{array}$ & 33,943 & 2.70 & .55 & -1 & 38 & 11 & .53 & .42 \\
\hline L amygdala & 384 & 2.30 & .46 & -25 & -4 & -15 & .25 & .13 \\
\hline $\mathrm{R}$ amygdala & 1,134 & 2.88 & .59 & 26 & -1 & -17 & .24 & .11 \\
\hline L putamen/globus pallidus & 2,556 & 2.59 & .53 & -19 & 4 & 6 & .28 & .15 \\
\hline $\mathrm{R}$ putamen/globus pallidus & 3,661 & 2.50 & .51 & 20 & 5 & 1 & .31 & .18 \\
\hline L caudate & 2,278 & 2.68 & .54 & -10 & 0 & 11 & .40 & .33 \\
\hline $\mathrm{R}$ caudate & 3,062 & 2.80 & .57 & 13 & -2 & 16 & .37 & .26 \\
\hline
\end{tabular}

Note. $\mathrm{L}=$ left. $\mathrm{R}=$ right. $\mathrm{M}=$ medial. $\mathrm{OFC}=$ orbitofrontal cortex. $\mathrm{IFG}=$ inferior frontal gyrus. $\mathrm{ACC}=$ anterior cingulate cortex. $\mathrm{dACC}=$ dorsal $\mathrm{ACC}$. $\mathrm{gACC}=$ genual $\mathrm{ACC} . \mathrm{PCC}=$ posterior cingulate cortex. $\mathrm{NAc}=$ nucleus accumbens. $\mathrm{BA}=$ Brodmann's Area. Location $=$ coordinates are for the maximum $z$ value and are for MNI152 2009 space, with the $x$ axis moving from left to right. Inc $\mathrm{r}=$ correlation with DLPFC seed cluster time series during the incongruent condition.

Con $\mathrm{r}=$ correlation with DLPFC seed cluster time series during the congruent condition.

${ }^{a}$ Cluster did not survive when only using the unique variance associated with the seed cluster.

temperament types. Finally, the cluster associated with only approach exhibited greater condition-dependent connectivity with anterior-medial OFC than did the cluster associated with only avoidance.

The DLPFC cluster associated with both temperament types exhibited stronger condition-dependent connectivity with dACC/ gACC/paracingulate than did either of the other DLPFC clusters. The cluster associated with both temperament types exhibited more condition-dependent connectivity with PCC than the cluster associated only with approach. However, the opposite pattern held for the cluster associated with only avoidance, with this cluster exhibiting greater condition-dependent connectivity with $\mathrm{PCC} /$ precuneus than did the cluster associated with both temperament types (although these clusters did not overlap).

With regard to BG, the cluster associated with both temperament types exhibited greater condition-dependent connectivity with right caudate than both of the other DLPFC clusters. No other differences emerged in BG.

Moderation of PPI connectivity by temperamental motivation. Table 5 lists regions in which motivational temperament factor scores moderated task-condition-dependent connectiv- ity with DLPFC clusters. The approach/avoidance sum score was associated with increased condition-dependent connectivity between left agranular OFC/IFG/insula and the left DLPFC cluster associated with both approach and avoidance.

The approach temperament score was associated with increased condition-dependent connectivity between the left DLPFC cluster associated only with approach and both right lateral OFC and left putamen/globus pallidus. Additionally, the approach temperament score was associated with increased connectivity between the left DLPFC cluster associated only with approach and anterior-medial OFC, although this cluster did not survive cluster thresholding. The avoidance temperament score did not significantly moderate condition-dependent connectivity with the left DLPFC cluster associated only with approach.

The avoidance temperament score was associated with increased condition-dependent connectivity between the right DLPFC cluster associated only with avoidance and both anteriormedial OFC/frontal pole and left agranular/lateral OFC. Unexpectedly, the approach temperament score was associated with decreased condition-dependent connectivity between the right DLPFC cluster associated only with avoidance and medial dACC/ gACC/paracingulate, right amygdala, and right putamen/NAc. 


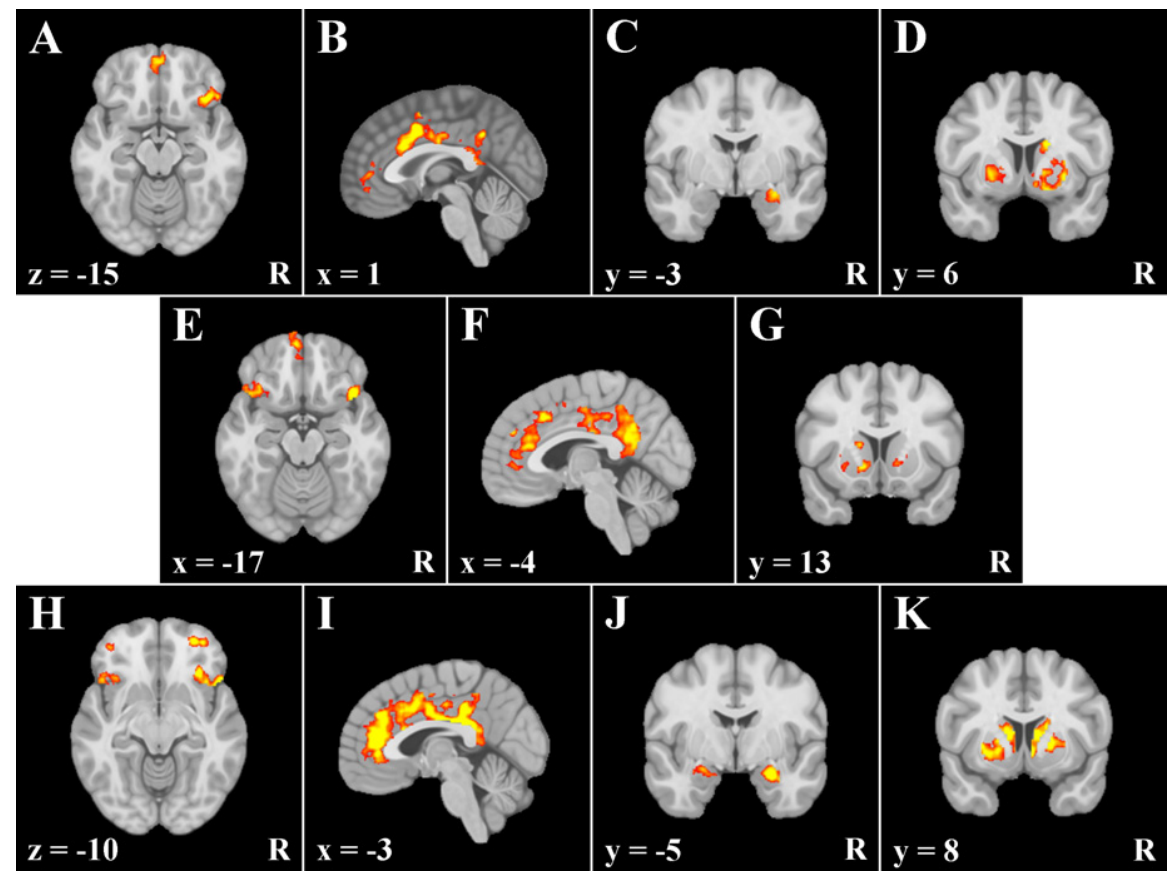

Figure 2. Areas exhibiting condition dependent correlations with DLPFC clusters in sample 2. $\mathrm{R}=$ right. $\mathrm{x}, \mathrm{y}$, and $\mathrm{z}=$ coordinates in MNI 2009a space. A-D =clusters exhibiting greater connectivity with the left DLPFC cluster associated with approach temperament. E-G = clusters exhibiting greater connectivity with the right DLPFC cluster associated with avoidance temperament. $\mathrm{H}-\mathrm{K}=$ clusters exhibiting greater connectivity with the left DLPFC cluster associated with approach and avoidance temperament.

\section{Discussion}

Across two independent samples, present findings supported the hypothesis that activity in regions of DLPFC associated with trait approach and avoidance motivation would exhibit greater positive correlations with activity in orbitofrontal cortex, anterior cingulate, amygdala, and basal ganglia when goal maintenance was challenged. Although there were differences in findings across samples, conjunction analyses revealed a consistent set of regions that exhibited connectivity with each DLPFC cluster.

As hypothesized, when goal maintenance was challenged, all three DLFPC clusters exhibited increased connectivity with the area of ACC thought to be involved in maintaining the average value of actions (Rushworth \& Behrens, 2008). The fact that this area of ACC exhibited increased connectivity with all three DLPFC

Table 3. Areas Exhibiting Effects in the Conjunction of Sample 1 and Sample 2

\begin{tabular}{|c|c|c|c|c|c|}
\hline \multirow[b]{2}{*}{ Region } & \multirow[b]{2}{*}{ Cluster size $\left(\mathrm{mm}^{3}\right)$} & \multirow[b]{2}{*}{ Mean $z$ value } & \multicolumn{3}{|c|}{ Location } \\
\hline & & & $\mathrm{X}$ & $\mathrm{Y}$ & $\mathrm{Z}$ \\
\hline \multicolumn{6}{|l|}{ L DLPFC associated with approach temperament } \\
\hline $\mathrm{R}$ agranular/lateral OFC (BA 11/47) & 1,223 & 2.04 & 19 & 5 & -18 \\
\hline M dACC/paracingulate (BA 6/24/32) & 3,258 & 2.06 & 1 & 19 & 27 \\
\hline $\mathrm{R}$ amygdala & 338 & 2.18 & 23 & 1 & -15 \\
\hline L putamen/globus pallidus & 1,987 & 2.05 & -24 & 8 & -6 \\
\hline R putamen & 1,385 & 2.42 & 21 & 4 & -13 \\
\hline \multicolumn{6}{|l|}{ R DLPFC associated with avoidance temperament } \\
\hline L agranular OFC (BA 11/47) & 1,442 & 2.09 & -42 & 20 & -17 \\
\hline M dACC/gACC/paracingulate (BA 6/9/24/32) & 9,379 & 2.12 & -1 & 29 & 17 \\
\hline M PCC/precuneus (BA $7 / 23 / 29 / 31$ ) & 5,702 & 2.14 & -4 & -52 & 35 \\
\hline $\mathrm{R}$ putamen/globus pallidus & 2,387 & 2.02 & 32 & 3 & 5 \\
\hline \multicolumn{6}{|l|}{ L DLPFC associated with approach and avoidance temperament } \\
\hline R agranular OFC/IFG/insula (BA 11/13/47) & 2,739 & 2.19 & 45 & 17 & -13 \\
\hline $\mathrm{M} \mathrm{dACC} / \mathrm{gACC} /$ paracingulate gyrus (BA $6 / 9 / 24 / 32 / 33$ ) & 15,316 & 2.20 & 3 & 39 & 16 \\
\hline M PCC (BA 23/29/31) & 1,394 & 2.10 & 2 & -37 & 24 \\
\hline M PCC/precuneus (BA 7/23/31) & 1,302 & 2.08 & 6 & -43 & 49 \\
\hline $\mathrm{L}$ amygdala & 380 & 2.11 & -23 & -4 & -16 \\
\hline $\mathrm{R}$ putamen/globus pallidus/caudate & 2,373 & 2.27 & 13 & 3 & 13 \\
\hline
\end{tabular}

Note $. \mathrm{L}=$ left. $\mathrm{R}=$ right. $\mathrm{M}=$ medial. $\mathrm{OFC}=$ orbitofrontal cortex. $\mathrm{IFG}=$ inferior frontal gyrus. $\mathrm{AC}=$ anterior cingulate cortex. $\mathrm{dACC}=$ dorsal $\mathrm{ACC}$. $\mathrm{gACC}=$ genual ACC. $\mathrm{PCC}=$ posterior cingulate cortex. BA = Brodmann's Area. Location $=$ coordinates are for the maximum $z$ value and are for MNI152 2009 space, with the $x$ axis moving from left to right. 
Table 4. Areas Exhibiting Differences in Condition-Dependent Correlations with DLPFC Clusters

\begin{tabular}{|c|c|c|c|c|c|c|c|}
\hline \multirow[b]{2}{*}{ Region } & \multirow{2}{*}{$\begin{array}{l}\text { Direction of } \\
\text { difference }\end{array}$} & \multirow[b]{2}{*}{ Cluster size $\left(\mathrm{mm}^{3}\right)$} & \multirow[b]{2}{*}{ Mean $z$ value } & \multirow[b]{2}{*}{ Cohen's $d$} & \multicolumn{3}{|c|}{ Location } \\
\hline & & & & & $\mathrm{X}$ & $\mathrm{Y}$ & $\mathrm{Z}$ \\
\hline \multicolumn{8}{|l|}{ L DLPFC associated with approach vs. R DLPFC associated with avoidance } \\
\hline $\mathrm{M}$ anterior OFC (BA 11) & Positive & 1,225 & 2.08 & .31 & 6 & 48 & -30 \\
\hline \multicolumn{8}{|c|}{ L DLPFC associated with approach vs. L DLPFC associated with approach and avoidance } \\
\hline $\mathrm{M}$ anterior OFC (BA 11) & Negative & 3,325 & -2.23 & .30 & 1 & 47 & -31 \\
\hline L agranular OFC/posterior-middle OFC (BA 11) & Negative & 1,243 & -2.27 & .24 & -20 & 21 & -27 \\
\hline M dACC/gACC/paracingulate (BA 6/8/24/32) & Positive & 6,335 & 2.87 & .27 & 11 & 33 & 24 \\
\hline M PCC (BA 23/31) & Positive & 1,099 & 2.40 & .24 & 3 & -27 & 28 \\
\hline $\mathrm{R}$ caudate & Positive & 965 & 2.87 & .19 & 8 & 7 & 9 \\
\hline \multicolumn{8}{|c|}{ R DLPFC associated with avoidance vs. L DLPFC associated with approach and avoidance } \\
\hline $\mathrm{M}$ anterior $\mathrm{OFC} /$ frontal pole/L anterior-middle OFC (BA 10/11) & Negative & 4,529 & -2.46 & .31 & -2 & 70 & 3 \\
\hline L lateral OFC (BA 11/47) & Negative & 1,608 & -2.16 & .18 & -48 & 46 & -16 \\
\hline $\mathrm{M}$ dACC/gACC/paracingulate (BA 8/24/32) & Positive & 3,544 & 2.76 & .26 & 9 & 34 & 32 \\
\hline M PCC/precuneus (BA 7/23/31) & Negative & 1,952 & -2.84 & .29 & -3 & -53 & 35 \\
\hline $\mathrm{R}$ caudate & Positive & 1,189 & 2.88 & .26 & 8 & 11 & 9 \\
\hline
\end{tabular}

Note. $\mathrm{L}=$ left. $\mathrm{R}=$ right. $\mathrm{M}=$ medial. $\mathrm{OFC}=$ orbitofrontal cortex. $\mathrm{ACC}=$ anterior cingulate cortex. $\mathrm{dACC}=$ dorsal $\mathrm{ACC} . \mathrm{gACC}=$ genual $\mathrm{ACC} . \mathrm{PCC}=$ posterior cingulate cortex. BA = Brodmann's Area. Location = coordinates are for the maximum $z$ value and are for MNI152 2009 space, with the $x$ axis moving from left to right.

clusters when goal maintenance was challenged suggests that it plays a central role in maintaining goal pursuit. In addition to this cingulate area, PCC and gACC consistently showed increased connectivity with the right DLPFC cluster and the left DLPFC cluster associated with both temperament types. Buckner and Carroll (2007) proposed that these areas of cingulate use past memories to generate potential future scenarios (termed prospection), aiding in the prediction of future events.

Prospection is essential to goal pursuit, because a representation of each potential outcome, based on past experience, is needed to evaluate the predicted value of that outcome. The ability to incorporate motivationally relevant information into anticipatory processing when considering a potential outcome will make that option seem more attractive (or unattractive if the outcome is unpleasant). Research suggests that PCC is involved in the incorporation of emotional and motivational aspects of memories into imagined scenarios (Maddock, 1999). This hypothesized role for PCC has been supported by several studies, including one that found more PCC activation when participants considered approach- and avoidance-related goals (Johnson et al., 2006). Additionally, a recent study found that PCC activation was associated with imagining potential future outcomes, and activation in PCC predicted both the reported value of a delayed reward option and the choice of this delayed reward over a smaller but less delayed reward (Peters \& Buchel, 2010). In summary, present findings suggest that DLPFC engages several areas of cingulate associated with different predictive functions to determine the best course of action.

Table 5. Areas in which Temperamental Motivation Moderates Condition-Dependent Correlations

\begin{tabular}{|c|c|c|c|c|c|c|c|}
\hline \multirow[b]{2}{*}{ Region } & \multirow[b]{2}{*}{ Cluster size $\left(\mathrm{mm}^{3}\right)$} & \multirow[b]{2}{*}{ Mean $z$ value } & \multirow[b]{2}{*}{$\Delta R^{2}$} & \multirow[b]{2}{*}{ Significant in Sample 1/2 } & \multicolumn{3}{|c|}{ Location } \\
\hline & & & & & $\mathrm{X}$ & $\mathrm{Y}$ & $\mathrm{Z}$ \\
\hline \multicolumn{8}{|l|}{ L DLPFC associated with approach temperament } \\
\hline \multicolumn{8}{|l|}{ Approach temperament } \\
\hline R lateral OFC (BA 11/47) & 728 & 2.01 & .06 & $\mathrm{Y} / \mathrm{Y}$ & 45 & 42 & -12 \\
\hline L putamen/globus pallidus & 764 & 2.05 & .05 & $\mathrm{Y} / \mathrm{Y}$ & -29 & -10 & -2 \\
\hline \multicolumn{8}{|l|}{ Avoidance temperament } \\
\hline- & - & - & - & - & - & - & - \\
\hline \multicolumn{8}{|l|}{$\mathrm{R}$ DLPFC associated with avoidance temperament } \\
\hline \multicolumn{8}{|l|}{ Approach temperament } \\
\hline M dACC/gACC/paracingulate (BA 6/24/32) & 3,124 & -2.41 & .10 & $\mathrm{Y} / \mathrm{Y}$ & -3 & -3 & 46 \\
\hline $\mathrm{R}$ amygdala & 947 & -2.65 & .06 & $\mathrm{Y} / \mathrm{N}$ & 26 & -1 & -26 \\
\hline $\mathrm{R}$ putamen/NAc & 1,773 & -2.53 & .11 & $\mathrm{Y} / \mathrm{N}$ & 27 & 14 & 1 \\
\hline \multicolumn{8}{|l|}{ Avoidance temperament } \\
\hline M anterior OFC/frontal pole (BA 10/11) & 841 & 2.14 & .06 & $\mathrm{~N} / \mathrm{Y}$ & 13 & 71 & -8 \\
\hline L agranular/lateral OFC (BA 11/47) & 715 & 2.06 & .06 & $\mathrm{Y} / \mathrm{Y}$ & -48 & 43 & -13 \\
\hline \multirow{2}{*}{\multicolumn{8}{|c|}{$\begin{array}{l}\text { L DLPFC associated with approach and avoidance temperament } \\
\text { Approach/avoidance temperament sum }\end{array}$}} \\
\hline & & & & & & & \\
\hline L agranular OFC/IFG/insula (BA 11/13/47) & 904 & 2.13 & .05 & $\mathrm{Y} / \mathrm{Y}$ & -51 & 23 & -3 \\
\hline
\end{tabular}

Note. $\mathrm{L}=$ left. $\mathrm{R}=$ right. $\mathrm{M}=$ medial. $\mathrm{OFC}=$ orbitofrontal cortex. $\mathrm{IFG}=$ inferior frontal gyrus. $\mathrm{ACC}=$ anterior cingulate cortex. $\mathrm{dACC}=$ dorsal $\mathrm{ACC}$. gACC $=$ genual ACC. $\mathrm{NAc}=$ nucleus accumbens. $\mathrm{BA}=$ Brodmann's Area. $\Delta R^{2}=$ the percen age of variance in condition-dependent connectivity accounted for by motivation score. Significant in Sample 1/2: $\mathrm{Y}=$ significant moderation was observed when examined in that sample, $\mathrm{N}=$ significant moderation was not observed when examined in that sample. Location: coordinates are for the maximum $z$ value and are for MNI152 2009 space, with the $x$ axis moving from left to right. 
Also in line with present hypotheses, both left DLPFC clusters consistently exhibited increased connectivity with amygdala when goal maintenance was challenged. This suggests that DLPFC receives information from amygdala, likely along with agranular OFC/insula, about what stimulus features are salient, and/or biasing which stimulus features are considered goal-relevant during the incongruent condition (biasing of stimulus features is not needed during the congruent condition).

All three DLPFC clusters consistently exhibited increased connectivity with regions of BG when goal maintenance was challenged, supporting present hypotheses. These included areas of putamen that research suggests influence action selection and preparation (Tremblay, Worbe, \& Hollerman, 2009), providing a means by which DLPFC may influence behavior. Finally, present findings supported the hypothesis that all three DLPFC clusters would exhibit increased connectivity with regions of OFC when goal maintenance was challenged. Furthermore, the pattern of connections between DLPFC and OFC that survived the conjunction analyses is consistent with the parcellation of OFC observed by Wager et al. (2008). Specifically, in the present study, left DLPFC was consistently connected to right lateral OFC, which Wager et al. found to be associated with pleasant valence, and right DLPFC was consistently connected to left lateral OFC, which Wager et al. found to be associated with unpleasant valence. However, it is important to note that, when looking beyond just those clusters surviving the conjunction analyses, the findings of the present study are less consistent with the pattern observed in Wager et al. For example, right DLPFC was associated with bilateral agranular OFC in Sample 2, rather than just left OFC.

These findings provide support for the hypothesis that regions of DLFPC thought to be involved in the integration of motivational and executive processes (Spielberg, Miller, et al., 2011; Spielberg et al., 2012) are part of a neural network involved in goal pursuit. Importantly, present findings indicate that each of the DLPFC clusters examined had an independent relationship with OFC, cingulate, amygdala, and BG (with the exception of the right DLPFC cluster associated with avoidance temperament and amygdala), given that these findings remained when only the unique variance associated with each cluster was used.

The fact that each DLPFC cluster was independently associated with the other brain regions in the network suggests that these regions of DLPFC play distinct roles in goal pursuit. This hypothesis was supported by analyses examining differential connectivity between DLPFC clusters and assessing moderation of connectivity by motivational temperament. For example, present findings suggest that network processing associated with the left and right DLPFC clusters related to approach and avoidance motivation, respectively, may be specific to the particular type of goal related to that cluster (i.e., approach or avoidance goals). Specifically, the left DLPFC cluster associated only with approach exhibited stronger connectivity with anterior-medial OFC than both of the other DLPFC clusters, and the approach temperament factor score was associated with increased connectivity between this DLPFC cluster and anterior-medial and right-lateral OFC (although the cluster in anterior-medial OFC did not survive cluster thresholding). These findings suggest that this DLPFC cluster has a specific role in the selection of approach goals based on stimulus reward values received from OFC and/or that this DLPFC cluster differentially modulates value information in these regions of OFC based on current goals.

Similarly, the right DLPFC cluster associated only with Avoidance Temperament exhibited stronger connectivity with left lateral
OFC (a region associated with the maintenance of punishment stimulus value; O'Doherty, 2007) than the cluster associated with both temperament types, and the avoidance temperament score was associated with increased connectivity between this cluster and left lateral/agranular OFC. These findings suggest that this DLPFC cluster has a specific role in the selection of avoidance goals based on stimulus punishment values received from left lateral OFC and/or that this DLPFC cluster differentially modulates value information in this region of OFC.

Finally, the DLPFC cluster associated with both temperament types exhibited stronger connectivity with dACC/gACC/ paracingulate than did the other DLPFC clusters, indicating that this DLPFC cluster has a specific role in the selection of appropriate action strategies. This hypothesis is also supported by the fact that this DLPFC cluster exhibited stronger connectivity with right caudate than did the other DLPFC clusters, given research suggesting that this caudate area is associated with action selection and preparation (Tremblay et al., 2009). In contrast, the left DLPFC cluster associated with both temperament types exhibited weaker connectivity with several areas of OFC (including anterior-middle OFC) than did the other clusters, suggesting that this DLPFC cluster is less involved in the selection of goals based on stimulus value and/or that this DLPFC cluster is not involved in the modulation of stimulus value information. This finding supports the hypothesis that this DLPFC cluster is involved in motivational processes that are independent of motivational direction. This may seem inconsistent with the present finding that the approach/ avoidance sum score was associated with increased connectivity between this DLPFC cluster and left agranular OFC/IFG/insula. However, research implicates agranular OFC, along with adjacent anterior insula, in parsing the most salient stimuli for current goals from all internal and external stimuli (e.g., Seeley et al., 2007) rather than the maintenance of stimulus value. Therefore, this DLPFC cluster may be receiving information about salience from this area and/or biasing what is identified as salient.

Unexpectedly, the Approach Temperament factor score was associated with decreased connectivity between the right DLPFC cluster associated with avoidance and a number of regions, including right amygdala. Although unexpected, these findings are consistent with previous conceptualizations of approach and avoidance motivational systems that posit mutual inhibition between the systems (e.g., Tomarken \& Keener, 1998). Additionally, this finding provides insight into why the right DLPFC cluster exhibited a weak and inconsistent relationship with amygdala in the present study. Specifically, present findings suggest that there is a relationship between these regions only when Approach Temperament is low.

An important general consideration for the present study is whether the color-word Stroop is potentially differentially relevant for approach or avoidance motivation. Specifically, it is possible that the particular processes isolated by the task contrast (ignoring color information that conflicts with the goal) are more central to, or heavily recruited by, one of the motivational systems. One possibility is that inhibiting incorrect responses is more relevant to the avoidance motivational system, given that ensuring that unwanted outcomes do not occur is a central aspect of this system (Elliot \& Covington, 2001). Avoidance may be active (engaging in behavior that prevents the undesired outcome from occurring) and/or it may involve inhibition of behavior until enough information is gained to appropriately guide responses (as in conceptualizations of the Behavioral Inhibition System; Gray, 1994). Therefore, inhibitory processes may be more often engaged as part of the avoidance system. 
Alternatively, it is possible that the task used in the present study may be more relevant to the approach motivational system. Specifically, research suggests that inhibition of incorrect responses by PFC is accomplished by the up-regulation of appropriate responses (through excitation of the neurons involved in the correct behavior) rather than the down-regulation of inappropriate responses (through inhibition of the neurons involved in the incorrect behavior; e.g., the top-down excitatory model; Herd, Banich, \& O'Reilly, 2006; Miller \& Cohen, 2001; see Berkman \& Lieberman, 2009, for a discussion of this model in the context of goal pursuit). Given that obtaining a desired state is the central focus of approach motivation, it seems plausible that brain regions involved in the up-regulation of behavior related to obtaining a desired state will be engaged more often as part of the approach motivational system.

In summary, it appears that a case could be made that the task used in the present study is more relevant for either of the motivational systems. Future research may be able to tease this apart by employing task manipulations that differentially engage one motivational system (e.g., engage state approach or avoidance motivation by giving rewards or punishments). For example, if connectivity patterns were found to be similar for approach motivation when state rewards were given (potentially changing in strength, but not in the pattern of connections), but differed for avoidance motivation when state punishments were given, this could indicate that the task is more relevant for approach motivation.

\section{Strengths and Limitations}

The present study benefited from the use of two independent samples that are each quite large for the fMRI literature. Additionally, an empirically based method was used to identify seed clusters involved in the integration of motivational and executive processes, which is likely to be a vital function for efficient goal pursuit. Limitations include the connectivity analysis method, which was correlational and cannot determine the presence or direction of causality. In addition, although there was a great deal of consistency in findings across the two samples, confirmed by the conjunction analyses, there were regions that emerged in one sample only or in nonoverlapping locations. This may indicate that effects in these areas are weak. Alternatively, this may reflect real differences between the samples, which varied in several ways, including average age. Future research should examine whether connectivity with DLPFC is dependent on factors such as age. Another limitation of the present study is that it examined only a subset of regions that may be involved in approach/avoidance goal pursuit, based on research indicating these regions are important for the specific aspect of approach/avoidance goal pursuit examined here. Future research should expand the network to include other relevant regions, perhaps depending on the specific aspect of goal pursuit examined.

Finally, although the large sample size is a strength of the present study, the use of this sample allowed us to identify effects that were relatively small in size. Specifically, although effect sizes for the initial PPI analyses were in the medium range (Cohen's $d=.46-.65$; see Tables 1 and 2), effect sizes were smaller for the connectivity comparisons between DLPFC clusters (Cohen's $d=.18-.31$; see Table 4 ) and the moderation of PPI by trait approach/avoidance $\left(\Delta R^{2}=.05-.11\right.$; see Table 5$)$. It is widely understood that small effects can have a meaningful impact on functioning. For example, these effects may exert a consistent effect on extended goal pursuit, in which case effects that are small at a given moment may accumulate over time, resulting in substantial differences over longer timescales. However, the degree to which these effects accumulate over time is not yet known.

The present study provides evidence for a network of brain regions instantiating approach and avoidance motivation and related goal pursuit. Distinct areas of DLPFC associated with the integration of motivational and executive processes exhibited increased connectivity with OFC, ACC, amygdala, and BG when goal maintenance was challenged, supporting the hypothesis that these areas are part of a network supporting key aspects of approach and avoidance goal pursuit. Identification of this network moves research on the neural mechanisms of approach and avoidance motivation beyond a focus on single brain regions, paving the way for an understanding of the more complex processes involved in approach and avoidance motivation.

\section{References}

Adolphs, R., Tranel, D., Damasio, H., \& Damasio, A. R. (1995). Fear and the human amygdala. The Journal of Neuroscience, 15, 58795891.

Amiez, C., \& Petrides, M. (2009). Feedback valuation processing within the prefrontal cortex. In J. C. Dreher \& L. Tremblay (Eds.), Handbook of reward and decision making (pp. 213-228). London: Academic Press.

Andersson, J. L. R., Jenkinson, M., \& Smith, S. (2007). Non-linear optimization (Tech. Rep. TR07JA1). Oxford, United Kingdom: University of Oxford, FMRIB Centre.

Ballard, I. C., Murty, V. P., Carter, R. M., MacInnes, J. J., Heuttel, S. A., \& Adcock, R. A. (2011). Dorsolateral prefrontal cortex drives mesolimbic dopaminergic regions to initiate motivated behavior. Journal of Neuroscience, 31, 10340-10346. doi:10.1523/JNEUROSCI.089511.2011

Banich, M. T., Milham, M. P., Atchley, R., Cohen, N. J., Webb, A., Wszalek, T., . . Mangin, R. (2000). fMRI studies of Stroop tasks reveal unique roles of anterior and posterior brain systems in attentional selection. Journal of Cognitive Neuroscience, 12, 988-1000. doi:10.1162/ 08989290051137521

Beckmann, C., \& Smith, S. (2004). Probabilistic independent component analysis for functional magnetic resonance imaging. IEEE Transactions on Medical Imaging, 23, 137-152. doi:10.1109/TMI.2003.822821
Beckmann, M., Johansen-Berg, H., \& Rushworth, M. (2009). Connectivitybased parcellation of human cingulate cortex and its relation to functional specialization. Journal of Neuroscience, 29, 1175-1190. doi:10.1523/JNEUROSCI.3328-08.2009

Berkman, E. T., \& Lieberman, M. D. (2009). The neuroscience of goal pursuit: Bridging gaps between theory and data. In G. B. Moskowitz \& H. Grant (Eds.), The psychology of goals (pp. 98-126). New York: Guilford Press.

Berkman, E. T., \& Lieberman, M. D. (2010). Approaching the bad and avoiding the good: Lateral prefrontal cortical asymmetry distinguishes between action and valence. Journal of Cognitive Neuroscience, 22, 1970-1979. doi:10.1162/jocn.2009.21317

Bollen, K.A. (1989). Structural equations with latent variables. New York: John Wiley \& Sons.

Buckner, R. L., \& Carroll, D. C. (2007). Self-projection and the brain. Trends in Cognitive Sciences, 11, 49-57. doi:10.1016/j.tics.2006.11.004

Bush, G., Luu, P., \& Posner, M. I. (2000). Cognitive and emotional influences in anterior cingulate cortex. Trends in Cognitive Sciences, 4, 215-222. doi:10.1016/S1364-6613(00)01483-2

Bush, G., Vogt, B. A., Holmes, J., Dale, A. M., Greve, D., Jenike, M., \& Rosen, B. R. (2002). Dorsal anterior cingulate cortex: A role in rewardbased decision making. Proceedings of the National Academy of Sciences, USA, 99, 523-528. doi:10.1073/pnas.012470999 
Cain, C. K., \& LeDoux, J. E. (2008). Emotional processing and motivation: In search of brain mechanisms. In A. E. Elliot (Ed.), Handbook of approach and avoidance motivation (pp. 17-34). New York: Psychology Press.

Carter, C. S., Braver, T. S., Barch, D. M., Botvinick, M. M. Noll, D., \& Cohen, J. D. (1998). Anterior cingulate cortex, error detection, and the online monitoring of performance. Science, 280, 747-749. doi:10.1126/ science. 280.5364 .747

Carver, C. S., \& White, T. L. (1994). Behavioral inhibition, behavioral activation, and affective responses to impending reward and punishment: The BIS/BAS scales. Journal of Personality and Social Psychology, 67, 319-333. doi:10.1037/0022-3514.67.2.319

Compton, R. J., Banich, M. T., Mohanty, A., Milham, M. P., Herrington, J., Miller, G. A., ... Heller, W. (2003). Paying attention to emotion: An fMRI investigation of cognitive and emotional Stroop tasks. Cognitive, Affective \& Behavioral Neuroscience, 3, 81-96. doi:10.3758/ CABN.3.2.81

Costa, P. T., \& McCrae, R. R. (1992). Revised NEO personality inventory (NEOPI-R and Five Factor Inventory (NEO-FFI) professional manual. Odessa, FL: Psychological Assessment Resources.

Davidson, R. J., \& Irwin, W. (1999). The functional neuroanatomy of emotion and affective style. Trends in Cognitive Sciences, 3, 11-21. doi:10.1016/S0896-6273(04)00264-8

Dosenbach, N. U. F., Fair, D. A., Cohen, A. L., Schlaggar, B. L., \& Petersen, S. E. (2008). A dual-networks architecture of top-down control. Trends in Cognitive Sciences, 12, 99-105. doi:10.1016/ j.tics.2008.01.001

Elliot, A. J., \& Covington, M. V. (2001). Approach and avoidance motivation. Educational Psychology Review, 13, 73-92. doi:10.1023/ A:1009009018235

Elliot, A. J., \& Thrash, T. M. (2002). Approach-avoidance motivation in personality: Approach and avoidance temperaments and goals. Journal of Personality and Social Psychology, 82, 804-818. doi:10.1037/00223514.82.5.804

Elliott, R., Dolan, R. J., \& Frith, C. D. (2000). Dissociable functions in the medial and lateral orbitofrontal cortex: Evidence from human neuroimaging studies. Cerebral Cortex, 10, 308-317. doi:10.1093/cercor/ 10.3.308

Fonov, V. S., Evans, A. C., McKinstry, R. C., Almli, C. R., \& Collins, D. L. (2009). Unbiased nonlinear average age-appropriate brain templates from birth to adulthood. NeuroImage, 47, S102. doi:10.1016/S1053$8119(09) 70884-5$

Frith, C. D. (2000). The role of dorsolateral prefrontal cortex in the selection of action as revealed by functional imaging. In S. Monsell \& J. Driver (Eds.), Control of cognitive processes: Attention and performance XVIII (pp. 549-565). Cambridge, MA: MIT Press.

Gray, J. A. (1994). Three fundamental emotion systems. In P. Ekman \& R J. Davidson (Eds.), The nature of emotion (pp. 243-247). New York: Oxford University Press.

Haber, S. N. (2009). Anatomy and connectivity of the reward circuit. In J. C. Dreher \& L. Tremblay (Eds.), Handbook of reward and decision making (pp. 3-27). London: Academic Press.

Hare, T. A., Camerer, C. F., \& Rangel, A. (2009). Self-control in decisionmaking involves modulation of the vmPFC valuation system. Science, 324, 646-648. doi:10.1126/science. 1168450

Heller, W. (1993). Neuropsychological mechanisms of individual differences in emotion, personality, and arousal. Neuropsychology, 7, 476489. doi:10.1037/0894-4105.7.4.476

Herd, S. A., Banich, M. T., \& O'Reilly, R. C. (2006). Neural mechanisms of cognitive control: An integrative model of Stroop task performance and fMRI data. Journal of Cognitive Neuroscience, 18, 22-32. doi:10.1162/089892906775250012

Holland, P. C., \& Gallagher, M. (2004). Aygdala-frontal interactions and reward expectancy. Current Opinion in Neurobiology, 14, 148-155. doi:10.1016/j.conb.2004.03.007

Jenkinson, M., Bannister, P., Brady, M., \& Smith, S. (2003). Improved optimization for the robust and accurate linear registration and motion correction of brain images. NeuroImage, 17, 825-841. doi:10.1016/ S1053-8119(02)91132-8

Johnson, M., Raye, C., Mitchell, K., Touryan, S., Greene, E., \& NolenHoeksema, S. (2006). Dissociating medial frontal and posterior cingulate activity during self-reflection. Social Cognitive \& Affective Neuroscience, 1, 56-64. doi:10.1093/scan/ns1004

Knutson, B., Taylor, J., Kaufman, M., Peterson, R., \& Glover, G. (2005). Distributed neural representation of expected value. Journal of Neuroscience, 25, 4806-4812. doi:10.1523/JNEUROSCI.0642-05. 2005

Kouneiher, F., Charron, S., \& Koechlin, E. (2009). Motivation and cognitive control in the human prefrontal cortex. Nature Neuroscience, 12, 939-947. doi:10.1038/nn.2321

Kringelbach, M. L., \& Rolls, E. T. (2004). The functional neuroanatomy of the human orbitofrontal cortex: Evidence from neuroimaging and neuropsychology. Progress in Neurobiology, 72, 341-372. doi:10.1016/ j.pneurobio.2004.03.006

Lang, P. J., Bradley, M. M., \& Cuthbert, B. N. (1998). Emotion, motivation, and anxiety: Brain mechanisms and psychophysiology. Biological Psychiatry, 44, 1248-1263. doi:10.1037/0735-7044.112.5.1069

Lazar, M. A. (2008). The statistical analysis of functional MRI data. New York: Springer.

MacDonald, A. W., Cohen, J. D., Stenger, V. A., \& Carter, C. A. (2000). Dissociating the role of the dorsolateral prefrontal and anterior cingulate cortex in cognitive control. Science, 288, 1835-1838. doi:10.1126/ science.288.5472.1835

Maddock, R. J. (1999). The retrosplenial cortex and emotion: New insights from functional neuroimaging of the human brain. Trends in Neurosciences, 22, 310-316. doi:10.1016/S0166-2236(98)01374-5

Miller, E. K., \& Cohen, J. D. (2001). An integrative theory of prefrontal cortex function. Annual Review of Neuroscience, 24, 167-202. doi:10.1146/annurev.neuro.24.1.167

Nichols, T., Brett, M., Andersson, J., Wager, T., \& Poline, J. B. (2005). Valid conjunction inference with the minimum statistic. NeuroImage, 25, 653-660. doi:10.1016/j.neuroimage.2004.12.005

O'Doherty, J. P. (2007). Lights, camembert, action! The role of human orbitofrontal cortex in encoding stimuli, rewards, and choices. Annals or the New York Academy of Sciences, 1121, 254-272. doi:10.1196/ annals.1401.036

O'Doherty, J. P., \& Dolan, R. J. (2006). The role of human orbitofrontal cortex in reward prediction and behavioral choice: Insights from neuroimaging. In D. H. Zald \& S. L. Rauch (Eds.), The orbitofrontal cortex (pp. 265-284). Oxford, United Kingdom: Oxford University Press.

Pessoa, L., \& Adolphs, R. (2010). Emotion processing and the amygdala: From a "low road" to "many roads" of evaluating biological significance. Nature Reviews Neuroscience, 11, 773-782. doi:10.1038/ nrn2920

Peters, J., \& Buchel, C. (2010). Episodic future thinking reduces reward delay discounting through an enhancement of prefrontal-mediotemporal interactions. Neuron, 66, 138-148. doi:10.1016/j.neuron.2010.03.026

Porcelli, A. J., \& Delgado, M. R. (2009). Reward processing in the human brain: Insights from fMRI. In J. C. Dreher \& L. Tremblay (Eds.), Handbook of reward and decision making (pp. 165-184). London: Academic Press.

Rangel, A., \& Hare, T. (2010). Neural computations associated with goaldirected choice. Current Opinion in Neurobiology, 20, 262-270. doi:10.1016/j.conb.2010.03.001

Rushworth, M., \& Behrens, T. (2008). Choice, uncertainty and value in prefrontal and cingulate cortex. Nature Neuroscience, 11, 389-397. doi:10.1038/nn2066

Rushworth, M., Behrens, T., Rudebeck, P. H., \& Walton, M. E. (2007). Contrasting roles for the cingulate and orbitofrontal cortex in decisions and social behavior. Trends in Cognitive Sciences, 11, 168-176. doi:10.1016/j.tics.2007.01.004

Sabatinelli, D., Bradley, M. M., Fitzsimmons, J. R., \& Lang, P. J. (2005). Parallel amygdala and inferotemporal activation reflect emotional intensity and fear relevance. NeuroImage, 24, 1265-1270. doi:10.1016/ j.neuroimage.2004.12.015

Sakagami, S., \& Watanabe, M. (2007). Integration of cognitive and motivational information in the primate lateral prefrontal cortex. Annals of the New York Academy of Sciences, 1104, 89-107. doi:10.1196/ annals. 1390.010

Seeley, W. W., Menon, V., Schatzberg, A. F., Keller, J., Glover, G. H., Kenna, H., . . . Greicius, M. D. (2007). Dissociable intrinsic connectivity networks for salience processing and executive control. Journal of Neuroscience, 27, 2349-2356. doi:10.1523/JNEUROSCI.558706.2007

Spielberg, J. M., Heller, W., Silton, R. L., Stewart, J. L., \& Miller, G. A. (2011). Approach and avoidance profiles distinguish dimensions of anxiety and depression. Cognitive Therapy and Research, 35, 359-371. doi:10.1007/s10608-011-9364-0

Spielberg, J. M., Miller, G. A., Engels, A. S., Herrington, J. D., Sutton, B. P., Banich, M. T., \& Heller, W. (2011). Trait approach and avoidance 
motivation: Lateralized neural activity associated with executive function. NeuroImage, 54, 661-670. doi:10.1016/j.neuroimage.2010. 08.037

Spielberg, J. M., Miller, G. A., Warren, S. L., Engels, A. S., Sutton, B. P., $\&$ Heller, W. (2012). Trait motivation moderates neural activation associated with goal pursuit. Cognitive, Affective, \& Behavioral Neuroscience, 12, 308-322. doi:10.3758/s13415-012-0088-8

Spielberg, J. M., Stewart, J. L., Levin, R. L., Miller, G. A., \& Heller, W. (2008). Prefrontal cortex, emotion, and approach/withdrawal motivation. Social and Personality Psychology Compass, 2, 135-153. doi:10.1111/j.1751-9004.2007.00064.x

Staudinger, M. R., Erk, S., \& Walter, H. (2011). Dorsolateral prefrontal cortex modulates striatal reward encoding during reappraisal of reward anticipation. Cerebral Cortex, 21, 2578-2588. doi:10.1093/cercor/ bhr041

Stroop, J. R. (1935). Studies of interference in serial verbal reactions. Journal of Experimental Psychology: General, 18, 643-662. doi:10.1037/h0054651

Szatkowska, I., Bogorodzki, P., Wolak, T., Marchewka, A., \& Szeszkowski, W. (2008). The effect of motivation on working memory: An fMRI and SEM study. Neurobiology of Learning and Memory, 90, 475-478. doi:10.1016/j.nlm.2008.06.001

Taylor, S. F., Welsh, R. C., Wager, T. D., Phan, K. L., Fitzgerald, K. D., \& Gehring, W. J. (2004). A functional neuroimaging study of motivation and executive function. NeuroImage, 21, 1045-1054. doi:10.1016/ j.neuroimage.2003.10.032

Tomarken, A. J., \& Keener, A. D. (1998). Frontal brain asymmetry and depression: A self-regulatory perspective. Cognition and Emotion, 12, 387-420. doi:10.1080/026999398379655
Tremblay, L., Worbe, Y., \& Hollerman, J. R. (2009). The ventral striatum: A heterogenous structure involved in reward processing, motivation, and decision-making. In J. C. Dreher \& L. Tremblay (Eds.), Handbook of reward and decision making (pp. 51-77). London: Academic Press.

Tricomi, E. M., Delgado, M. R., \& Fiez, J. A. (2004). Modulation of caudate activity by action contingency. Neuron, 41, 281-292. doi:10.1016/S0896-6273(03)00848-1

Wager, T. D., Feldman Barrett, L., Bliss-Moreau, E., Lindquist, K. A., Duncan, S., Kober, H., ... Mize, J. (2008). The neuroimaging of emotion. In M. Lewis, J. M. Haviland-Jones, \& L. Feldman-Barrett (Eds.), Handbook of emotions (3rd ed., pp. 272-290). New York: Guilford Press.

Wager, T. D., \& Smith, E. E. (2003). Neuroimaging studies of working memory: A meta-analysis. Cognitive, Affective, \& Behavioral Neuroscience, 3, 255-274. doi:10.3758/CABN.3.4.255

Ward, D. B. (2000). Simultaneous inference for FMRI data. Retrieved from http://afni.nimh.nih.gov./pub/dist/doc/manual/AlphaSim.pdf

Watson, D., \& Clark, L. A. (1993). Behavioral disinhibition versus constraint: A dispositional perspective. In D. M. Wegner \& J. W. Pennebaker (Eds.), Handbook of mental control (pp. 506-527). New York: Prentice Hall.

Woolrich, M. W., Ripley, B. D., Brady, M., \& Smith, S. M. (2001). Temporal autocorrelation in univariate linear modeling fMRI data. NeuroImage, 14, 1370-1386. doi:10.1006/nimg.2001.0931

(ReceIVEd December 6, 2011; ACCEPTED June 13, 2012) 